\title{
Fabrication of dissolvable, polymer microneedles by through-hole mold filling
}

\begin{abstract}
The dissolvable, biocompatible polymer microneedles (MNs) offer competitive advantages over those made of other materials, e.g. added functionality, active and controlled release, improved resistance to shear-induced breakage, cost effectiveness, and additional safety, etc. Although casting or centrifugation is widely used to fabricate polymer MNs and other alternatives have been demonstrated, they are either time-consuming, relatively tedious or involved in complicated procedure. Here, we proposed a novel technique by filling the through-hole mold with polymer solution in conjunction with heating. The filter paper was used as the patch material. The results showed that the polyvinyl- pryrrolidone (PVP) microneedles can be obtained around 30 mins at $65^{\circ} \mathrm{C}$ heating temperature. The method is relatively simple, easy, low-cost and reliable, which has great potential for mass production.
\end{abstract}

Volume 6 Issue 3 - 2020

\author{
Chuan-I Chen,Yi-Je Juang \\ Department of Chemical Engineering, National Cheng Kung \\ University, Taiwan
}

\author{
Correspondence: Yi-Je Juang, Department of Chemical \\ Engineering, National Cheng Kung University, No. I University \\ Road,Tainan, Taiwan 7010I, Email yjjung@mail.ncku.edu.tw
}

Received: August 25, 2020 | Published: September 07, 2020

Keywords: microneedle, transdermal drug delivery, filter paper, polydimethylsiloxane, polyvinylpryrrolidone

\section{Introduction}

Transdermal drug delivery (TDD) has become an attractive approach for drug administration owing to its characteristic advantages such as prevention of the drug from degradation in the gastrointestinal tract and the first pass metabolism, sustaining the therapeutic drug level, non-invasive drug application, lower risk of infection, self-administration and improved patient compliance. ${ }^{1-3}$ TDD is a self-contained, discrete dosage form, which delivers the drug through the skin at controlled rate to the systemic circulation.

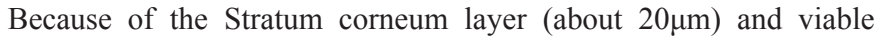
epidermis layer (about $300 \mu \mathrm{m}$ ) of human skin, penetrating these barriers is the first step for successful TDD. There are primarily two strategies applied to overcome the skin barrier. ${ }^{4}$ One is the chemical treatment where the chemical compounds like sulphoxides, azones, pyrrolidones, alcohols, alkanols, etc. are used to reversibly change the physical properties of lipid bilayer. The other is the physical treatment where the methods like iontophoresis, electroporation, ultrasound technique, photoacoustic approaches and microneedles are applied to create the reversible pinholes to allow the molecular drug to penetrate skin barrier. Among these techniques, application of microneedles (MNs) permits easy and painless administration without the need of expensive equipment and system integration. There are a variety of materials used to fabricate MNs such as silicon, ${ }^{5,6}$, metal,,${ }^{7,8}$ photoresist ${ }^{9}$ and polymers. ${ }^{10}$ However, concerns are raised due to risk of residual fragment caused by the broken MNs, biohazardous sharps waste and insufficient mechanical strength. To resolve these issues, the dissolvable and biodegradable polymers were adopted and many fabrication techniques have been proposed and demonstrated. ${ }^{11-19}$ For example, the droplet-born air blowing is a method that droplets of polymer solution are first dispensed on a substrate. Next, another substrate is brought into contact with the droplets and then slowly moved apart. Meanwhile, the air is blown to dry the solution. Once the solution is dried, the polymer forms hourglass like structures, which subsequently break into cone-like MNs. With this method, an array of MNs can be fabricated within 10 mins. ${ }^{13}$ The drawing method is another additive technique to produce MNs from 2D planar substrate. ${ }^{14}$ The MNs are formed by well-controlled, set temperatures at each individual steps starting from pillar contact with polymer substrate, followed by drawing of viscous polymer and solidification of polymer to separation. For the membrane filtration technique, a PDMS through-hole mold was used and formation of MNs followed the principle of cake formation during filtration and the MNs can be made within $1 \mathrm{hr} .^{18}$ Fabrication of MNs by 3D printing, scanning-based stereolithography (SLA) was proposed in recent years. ${ }^{19}$ The MN mold was first constructed by printing out the UVcurable resin and using a laser to track and "draw" each layer where the resin is cured as it travels along the $x-y$ plane. The female mold was fabricated by soft lithography for subsequent usage. With this method, the high aspect-ratio sharp MNs with tip radii from 20-40 $\mu \mathrm{m}$ can be obtained. Although there are various fabrication techniques available, casting in conjunction with vacuum or centrifugation is most widely practiced. ${ }^{20-23}$ For casting method, foaming may occur during vacuum which leads to difficulties in filling the PDMS negative mold. Repetitive steps may be required to completely fill the mold. As to the centrifugation method, multiple recursive procedures of centrifugation, removing the solvent and scraping the polymer deposit on top of the mold are mandatory. In addition, the maximum area of the MNs negative mold is constrained by the diameter of centrifugal tube. Hence, development of simple and low cost fabrication techniques for the dissolvable and biodegradable polymer MNs is still needed. In this study, a new method was introduced to fabricate the dissolvable and biodegradable polymer MNs via through-hole mold filling, where the entire process can be completed around 30mins and the polymer MNs patch is flexible. Here, we performed the skin insertion test to demonstrate that the polymer MNs as fabricated possess sufficient mechanical strength for transdermal drug delivery.

\section{Materials and methods}

\section{Materials}

Polyvinylpyrrolidone (PVP) with molecular weight 40000, the chromatography paper (Whatman No. 1) and Rhodamine 6G (R6G) were purchased from Sigma-Aldrich, USA. Polydimethylsiloxane 
(PDMS) was purchased from Dow Corning, USA. The epoxy was purchased from Emperor Chemical, Taiwan.

\section{Construction of the through-hole $\mathrm{MN}$ mold}

To fabricate the through-hole, PDMS mold with funnel-like cavities, a metallic MN mold was first obtained through wire electrodischarged machining. ${ }^{22}$ The mold consists of an array of $11 \times 11$ pyramid-shaped MNs with height, base and tip-to-tip spacing with 600,300 and $600 \mu \mathrm{m}$, respectively. The PDMS with the ratio of base to curing agent 10:1 was well mixed, degassed, and gently poured onto the mold, followed by placing it in the oven with temperature set at $65^{\circ} \mathrm{C}$ for $4 \mathrm{hrs}$ and separating the cured PDMS from the MN mold to obtain the negative mold. Next, the epoxy resin was poured onto the negative mold and cured to obtain the epoxy MNs. The epoxy MNs mold was fixed onto the petri dish and a piece of plastic sheet and a metallic block were placed on top of the epoxy MNs. The PDMS was again gently poured into the petri dish till the mold was filled. The assembly was then placed in the oven with temperature set at $65^{\circ} \mathrm{C}$ for $4 \mathrm{hrs}$. After curing, the metallic block and plastic sheet were removed and the through-hole, PDMS mold was obtained by separating it from the epoxy MNs mold.

\section{Fabrication of PVP microneedles}

Figure 1 shows the procedure of fabricating the PVP MNs by filling the through-hole, PDMS mold. First, the mold was placed on top of a porous material (e.g. tissue, filter paper) with larger holes facing up, followed by dispensing the PVP solution (approximately $0.02 \mathrm{~g}$ ). The small holes were in contact with the porous material Another filter paper intended to serve as the support of the MNs was put on top of the PVP solution, followed by laying down a plastic block weighted around $0.5 \mathrm{~g}$. The assembly was then placed on the hot plate with temperature ranging from 35 to $65^{\circ} \mathrm{C}$ for 30 to $45 \mathrm{mins}$. After drying, the PVP MNs with the filter paper was separated from the mold.

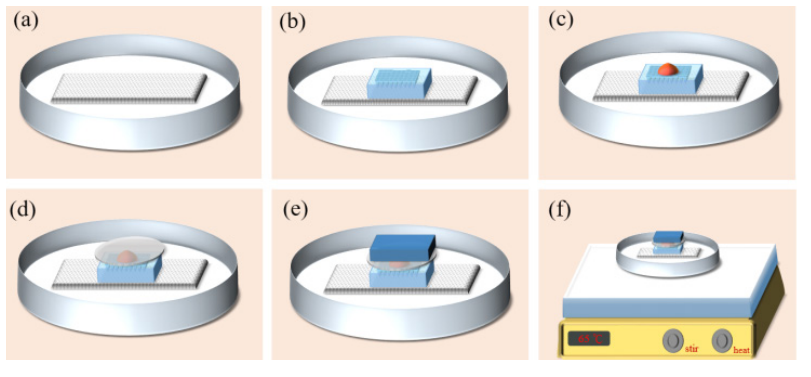

Figure I The schematic of fabrication of microneedles by through-hole mold filling.

\section{In vitro skin insertion test}

To evaluate the in vitro skin insertion capability of the PVP microneedles, R6G-loaded PVP microneedles were inserted into porcine cadaver skin using a constant-force applicator with force approximately $12 \mathrm{~N} .{ }^{22}$ The base of the microneedles, i.e. the filter paper, was then removed after 10mins. The dissolution of the microneedles and the red spots on the skin were observed via a stereomicroscope (Leica, Nikon P6000). For clear image, water and ethanol were used to wash out the R6G remained on skin surface and then one droplet of blue dye was dispensed onto the skin.

\section{In vitro penetration depth}

To observe the penetration depth, the samples with inserted microneedles were dipped into formalin for 3 days, followed by making paraffin sections. With $40 \mu \mathrm{m}$ intervals, 6 paraffin sections of the hydrated pig skin sample were sliced (Shandon Cryotome E, Thermo Electron Corporation, USA). The paraffin sections were observed under the fluorescent microscope (IX-71, Olympus, Tokyo, Japan).

\section{Results and discussion}

\section{PVP microneedles}

Figure 2A shows the image of the MNs fabricated by the throughhole mold filling process. It can be seen that the tip diameter and base of the MNs were approximately 40 and $300 \mu \mathrm{m}$, respectively. The MN patch was magnified and the pyramidal shape of the MNs was observed as shown in Figure 2B, which resembles the metallic mold. The processing conditions for the above MNs patch were as follows: $60 \mathrm{wt} \%$ PVP solution, $65^{\circ} \mathrm{C}$ drying temperature and $30 \mathrm{mins}$ drying time. A series of parametric studies was carried out as shown in Table 1. It can be seen that the drying temperature, the drying time and the solution concentration played important roles in formation of the MNs patch. Note that there were two scenarios for failing to form MNs. One is that the PVP MNs did not completely solidify after drying, which were destroyed when separating it from the mold. The other is that the cavities were not completely filled with the PVP solution, which led to broken or hollow MNs. The mechanism for formation of MNs patch was hypothesized as follows. After dispensing the PVP solution on top of the mold, followed by placing a filter paper and a piece of plastic block on top of the solution, there could be three directions for the fluid flow as shown schematically in Figure 3. One is the flow into the cavities, another is the flow outward and the other is the flow into the filter paper. Although the flow resistance is the largest in the direction into the filter paper owing to its smallest pore diameter (approximately $20 \mu \mathrm{m}$ compared to that of cavities and the gap between filter paper and mold, which is around hundreds of micron), solution imbibition through filter paper will still occur due to capillarity. Most of the solution will flow either outward, into the cavities or both. For the outward flow, it will reach the edge of the filter paper and imbibe into the filter paper. For the flow into cavities, it will fill the cavities and reach the bottom of the cavities, which are in contact with another filter paper. The cake filtration then takes place such that the polymer deposits on the filter paper and the solvent (i.e. water) wicks into the filter paper via capillarity. Meanwhile, the assembly is placed on top of a hot plate and heating impacts the process in two ways. One is that the solution viscosity decreases, which facilitates in solution flow. The other is that the solvent evaporates to allow formation of MNs. Therefore, the effect of the abovementioned factors (i.e. solution viscosity and drying rate) needs to be considered simultaneously. For example, heating at higher temperature will benefit formation of MNs in terms of decreasing the solution viscosity for better fluidity and reducing the processing time. However, higher temperature may also result in incomplete filling of the cavities due to faster drying. Lower concentration will permit easier handling of solution and better fluid flow, however, MNs may not be formed within reasonable processing time due to difficulties in solution drying and less solid content in the solution. From Table 1, it can be seen that heating at $35^{\circ} \mathrm{C}$ did not lead to formation of solid MNs after $2 \mathrm{hrs}$. Increasing the heating temperature up to $60^{\circ} \mathrm{C}$ was able to construct the solid MNs when using the $60 \mathrm{wt} \%$ solution and the heating time was around 1 hr. Further increase of the heating temperature to $65^{\circ} \mathrm{C}$ reduced the heating time down to $30 \mathrm{mins}$. At $65^{\circ} \mathrm{C}, 50 \mathrm{wt} \%$ solution concentration and 40-min heating time did not lead to successful formation of MNs. As the heating temperature set to $70^{\circ} \mathrm{C}$, it failed to obtain MNs as 
shown in Figure 4. It is speculated that the solution dried so quickly before it could completely fill the cavities.
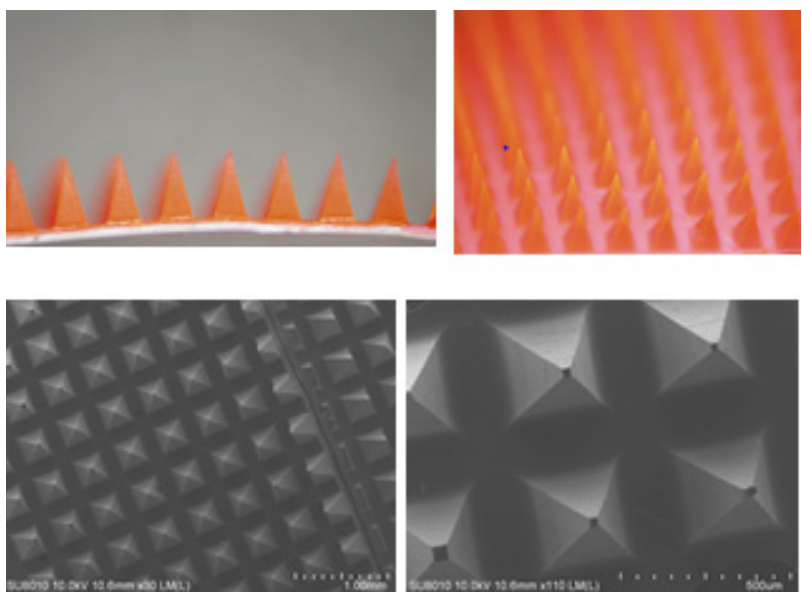

Figure 2 The image of the PVP microneedles patch taken by (a) optical microscope and (b) scanning electron microscope (SEM).

\section{Filter paper}
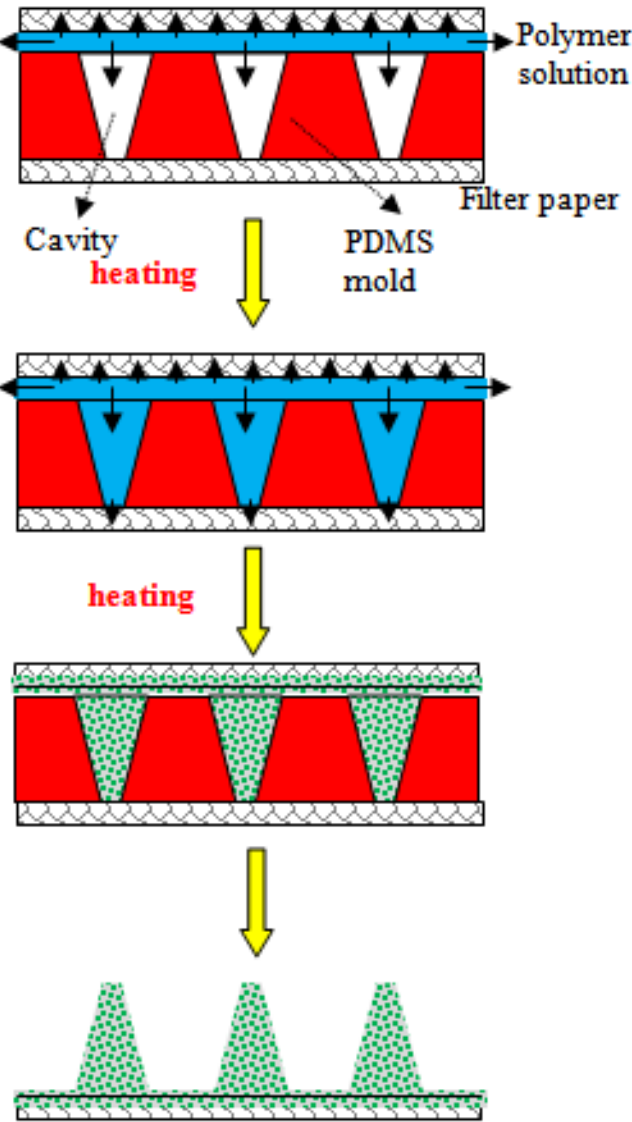

Figure 3 The hypothesized mechanism for MNs formation.
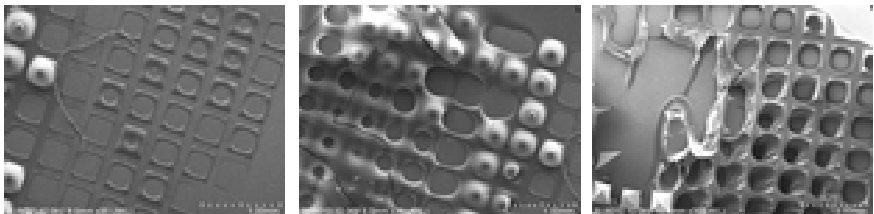

Figure 4 SEM images of the unsuccessful formation of MNs.
Table I The parametric study of the through-hole mold filling process

\begin{tabular}{llll}
\hline $\begin{array}{l}\text { Drying } \\
\text { temp. }\left({ }^{\circ} \mathbf{C}\right)\end{array}$ & $\begin{array}{l}\text { PVP conc. } \\
(\mathbf{w} / \mathbf{v} \%)\end{array}$ & $\begin{array}{l}\text { Drying time } \\
(\mathrm{min})\end{array}$ & $\begin{array}{l}\text { Formation of } \\
\text { MNs }\end{array}$ \\
\hline 35 & 40 & 130 & $\times$ \\
& 50 & 130 & $\times$ \\
60 & 60 & 130 & $\times$ \\
& 50 & 30 & $\times 1$ \\
65 & 60 & 60 & 0 \\
& 50 & 40 & $\times$ \\
& 60 & 30 & 0 \\
& 60 & 45 & 0
\end{tabular}

\section{Insertion test}

Figure 5 shows the insertion test using the MNs patch as fabricated. It can be seen that there were holes left on the pig skin after the support layer (i.e. filter paper) was removed. The penetration depth around 200 to $300 \mu \mathrm{m}$ was clearly observed as shown in Figure 6. This demonstrated that the MNs fabricated by the proposed method can be used just like those fabricated by other methods such as casting or centrifugal method.

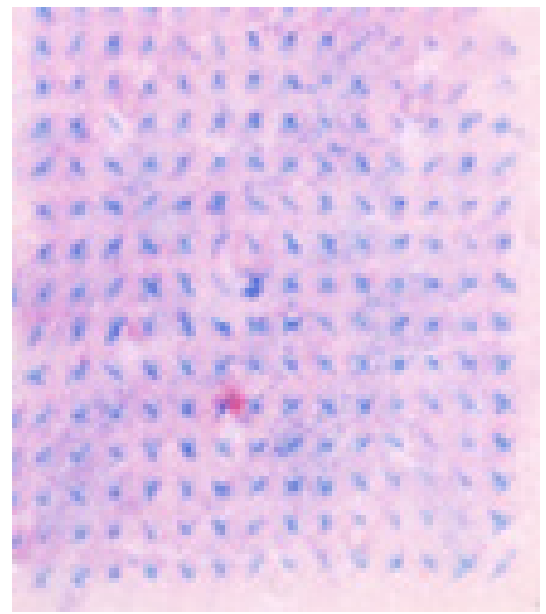

Figure $\mathbf{5}$ The insertion test of the pig skin using microneedle patch made by using $60 \%$ PVP solution and drying at $65^{\circ} \mathrm{C}$ for 30 mins.

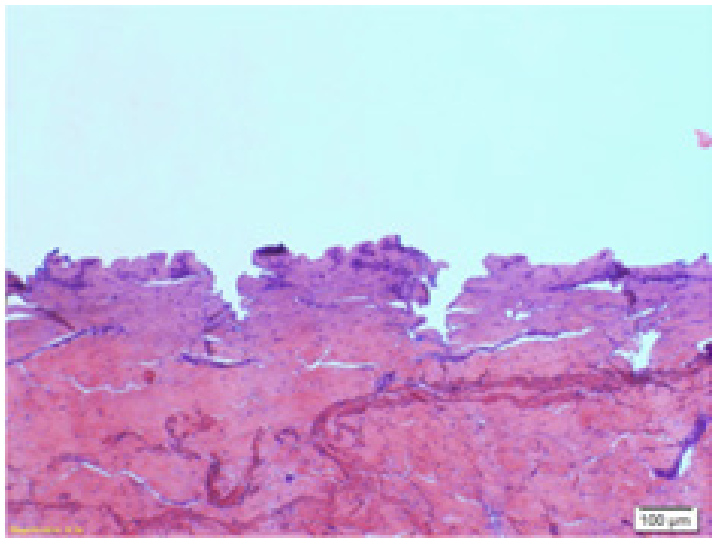

Figure 6 The cross-sectional view of the pig skin after insertion test. 


\section{Conclusions}

In this study, a new approach of fabricating dissolvable, polymer MNs, i.e. through-hole mold filling was proposed. A PDMS throughhole mold was first constructed, followed by placing a droplet of polymer solution on top of the mold and filter papers on both sides. The assembly was then placed on the hot plate. With this approach, the processing time can be reduced to $30 \mathrm{mins}$ when using $60 \%$ solution with heating temperature set at $65^{\circ} \mathrm{C}$. The approach is simple, relatively fast and low cost with equipment only involving a hot plate.

\section{Acknowledgments}

The authors would like to thank Prof. Mei-Chin Chen in Department of Chemical Engineering at National Cheng Kung University to provide the metallic MNs mold for replication and assistance in insertion tests. This work was supported by the Ministry of Science and Technology, Taiwan (MOST 107-2221-E-006-103-MY3).

\section{Conflicts of interest}

Authors declare that there is no conflict of interest.

\section{References}

1. Carl M Schoellhammer, Daniel Blankschtein, Robert Langer. Skin Permeabilization for Transdermal Drug Delivery: Recent Advances and Future Prospects. Expert Opin Drug Del. 2014;11(3):393-407.

2. Mark R Prausnitz, Robert Langer. Transdermal Drug Delivery. Nat Biotechnol. 2008;26(11):1261-1268.

3. Wijaya Martanto, Shawn $\mathrm{P}$ Davis, Nicholas $\mathrm{R}$ Holiday, et al Transdermal Delivery of Insulin Using Microneedles in vivo. Pharm Res. 2004;21(6):947-952.

4. Wijaya Martanto, Shawn P Davis, Nicholas R Holiday, et al. Penetration Enhancers. Adv Drug Deliver Rev. 2004;56(5):603-618.

5. Hamzah AA, Abd Aziz N, Majlis BY, et al. Optimization of HNA Etching Parameters to Produce High Aspect Ratio Solid Silicon Microneedles. J Micromech Microeng. 2012;22(9):095017.

6. Henry S, McAllister DV, Allen MG, et al. Microfabricated microneedles: A Novel Approach to Transdermal Drug Delivery. J Pharm Sci. 1998;87(8):922-925.

7. Po Chun C, Sheng Jen H, Chien Chon C, et al. A Three-Dimensional Enormous Surface Area Aluminum Microneedle Array with Nanoporous Structure. J Nanomater. 2013;164953.

8. Verbaan FJ, Bal SM, van den Berg DJ, et al. Improved piercing of microneedle arrays in dermatomed human skin by an impact insertion method. J Control Release. 2008;128(1):80-88.
9. Lee K, Lee HC, Lee DS, et al. Drawing Lithography: Three-Dimensional Fabrication of an Ultrahigh-Aspect-Ratio Microneedle. Adv Mater. 2010;22(4):483-486.

10. ciJuster, H. van der Aar, B., and de Brouwer, H., A Review on Microfabrication of Thermoplastic Polymer-Based Microneedle Arrays. Polymer Engineering and Sence. 2019;59(5):877-890.

11. Lee JW, Han MR, Park JH. Polymer Microneedles for Transdermal Drug Delivery. J Drug Target. 2013;21(3):211-223.

12. Park JH, Allen MG, Prausnitz MR. Biodegradable Polymer Microneedles: Fabrication, Mechanics and Transdermal Drug Delivery. J Control Release. 2005;104(1):51-66.

13. Kim JD, Kim M, Yang H, et al. Droplet-born Air Blowing: Novel Dissolving Microneedle Fabrication. J Control Release. 2013;170(3):430-436.

14. Lee K, Jung H. Drawing lithography for microneedles: A review of fundamentals and biomedical applications. Biomaterials. 2012;33:73097326.

15. Ito Y, Hagiwara E, Saeki A, et al. Feasibility of Microneedles for Percutaneous Absorption of Insulin. Eur J Pharm Sci. 2006;29(1):82-88.

16. Martin CJ, Allender CJ, Brain KR, et al. Low Temperature Fabrication of Biodegradable Sugar Glass Microneedles for Transdermal Drug Delivery Applications. J Control Release. 2012;158(1):93-101.

17. Moga KA, Bickford LR, Geil RD, et al. Rapidly-Dissolvable Microneedle Patches Via a Highly Scalable and Reproducible Soft Lithography Approach. Adv Mater. 2013;25(36):5060-5066.

18. Juang YJ, Deng YL, Lee IC. Membrane filtration: An unconventional route for fabrication of the flexible and dissolvable, polymer microneedle patches. Biomicrofluidics. 2016;10:044108.

19. Krieger, Nicky B, Manita D, et al. Simple and customizable method for fabrication of high-aspect ratio microneedle molds using low-cost 3D printing. Microsystems \& Nanoengineering. 2019;5:42.

20. Kochhar JS, Goh WJ, Chan SY, et al. A Simple Method of Microneedle Array Fabrication for Transdermal Drug. Delivery. Drug Dev Ind Pharm. 2013;39(2):299-309.

21. Lee JW, Park JH, Prausnitz MR. Dissolving Microneedles for Transdermal Drug Delivery. Biomaterials. 2008;29(13):2113-2124.

22. Ming-Hung L, Mei-Chin C. Dissolving Polymer Microneedle Patches for Rapid and Efficient Transdermal Delivery of Insulin to Diabetic Rats. Acta Biomater. 2013;9(11):8952-8961.

23. DeMuth PC, Garcia-Beltran WF, Ai-Ling ML, et al. Composite Dissolving Microneedles for Coordinated Control of Antigen and Adjuvant Delivery Kinetics in Transcutaneous Vaccination. Adv Funct Mater. 2013;23(2):161-172. 